\title{
A Rare Distant Metastasis of Papillary Cystadenocarcinoma Arising from Maxillary Gingiva
}

\author{
Fumihiro Nishimakia, b Takahiko Gibo ${ }^{a}$ b Keita Tsukadac \\ Takuro Noguchi $^{\text {b }}$ Toshirou Fukushima $^{\text {b }}$ Takashi Kobayashi ${ }^{b}$ \\ Nodoka Sekiguchib ${ }^{\text {Takesumi Ozawa }}{ }^{\text {b Tomonobu Koizumi }}{ }^{\text {b }}$ \\ aDepartment of Dentistry and Oral Surgery, Shinshu University School of Medicine, \\ Matsumoto, Japan; b Department of Comprehensive Cancer Therapy, Shinshu University \\ School of Medicine, Matsumoto, Japan; 'Department of Otorhinolaryngology, Shinshu \\ University School of Medicine, Matsumoto, Japan
}

\author{
Keywords \\ Chemotherapy - Low-grade malignancy - Minor salivary gland tumor - Pulmonary metastasis . \\ Salivary gland
}

\begin{abstract}
Papillary cystadenocarcinoma is an uncommon disease with low-grade histological and clinical features. Although the tumor has the potential to produce regional lymph node metastasis, there have been no reports of cases with distant metastasis. We describe a case of papillary cystadenocarcinoma arising from the maxilla that developed pulmonary metastasis 3 years after radical surgery of the primary tumor and regional lymph node. The histological findings were confirmed on resected specimens of the pulmonary nodule and a pathological diagnosis of a metastatic lesion derived from papillary cystadenocarcinoma was made. To our knowledge, this is the first report of the development of pulmonary metastasis in a patient with papillary cystadenocarcinoma. The present case suggests that papillary cystadenocarcinoma has the potential to produce lung metastasis in the clinical course. Based on our experience, we emphasize that long-term follow-up and/or careful examination are necessary in patients with cystadenocarcinoma, especially in patients with lymph node metastasis during the initial surgical therapy.




\section{Introduction}

Papillary cystadenocarcinoma is an uncommon malignant salivary gland tumor [1-3], sometimes involving the minor salivary gland [3-9]. Papillary cystadenocarcinoma is characterized macroscopically as a cystic and solid tumor with papillary growth. Histologically, the tumor is characterized by a predominantly cystic and invasive growth pattern with a papillary component. Vascular infiltration and perineural invasion are seldom observed [1, 2]. Cystadenocarcinoma shows indolent biological behavior and has low-grade histological features. The prognosis of this disease is excellent.

Although there have been several case reports of local recurrence after surgical resection and/or regional lymph node metastasis at initial presentation or during the clinical course [6-11], the progression of distant metastasis is poorly understood. We encountered an unusual case of papillary cystadenocarcinoma arising from the maxillary gingiva that developed pulmonary metastasis 3 years after radical surgery of the primary tumor and regional lymph node. The metastatic lesions were pathologically confirmed on resected pulmonary specimens. Here, we report the clinical course and present a review of the relevant literature.

\section{Case Presentation}

A 66-year-old man had noticed a painless mass in the left upper jaw and visited a local hospital. Intraorally, a well-circumscribed exophytic lesion measuring $2 \times 2 \mathrm{~cm}$ involving the left maxillary gingiva was noticed. The patient was referred to our hospital due to suspected papillary cystadenocarcinoma based on histological examination of the biopsy specimen. Computed tomography (CT) and magnetic resonance imaging (MRI) identified a solid mass $15 \mathrm{~mm}$ in diameter in the left upper jaw area (Fig. 1A, B). No regional lymph node metastases were detected on CT or MRI. In addition, neither systemic CT nor ${ }^{18} \mathrm{~F}$-fluorodeoxy glucose positron emission tomography (FDG-PET) revealed abnormal findings or uptake in both the
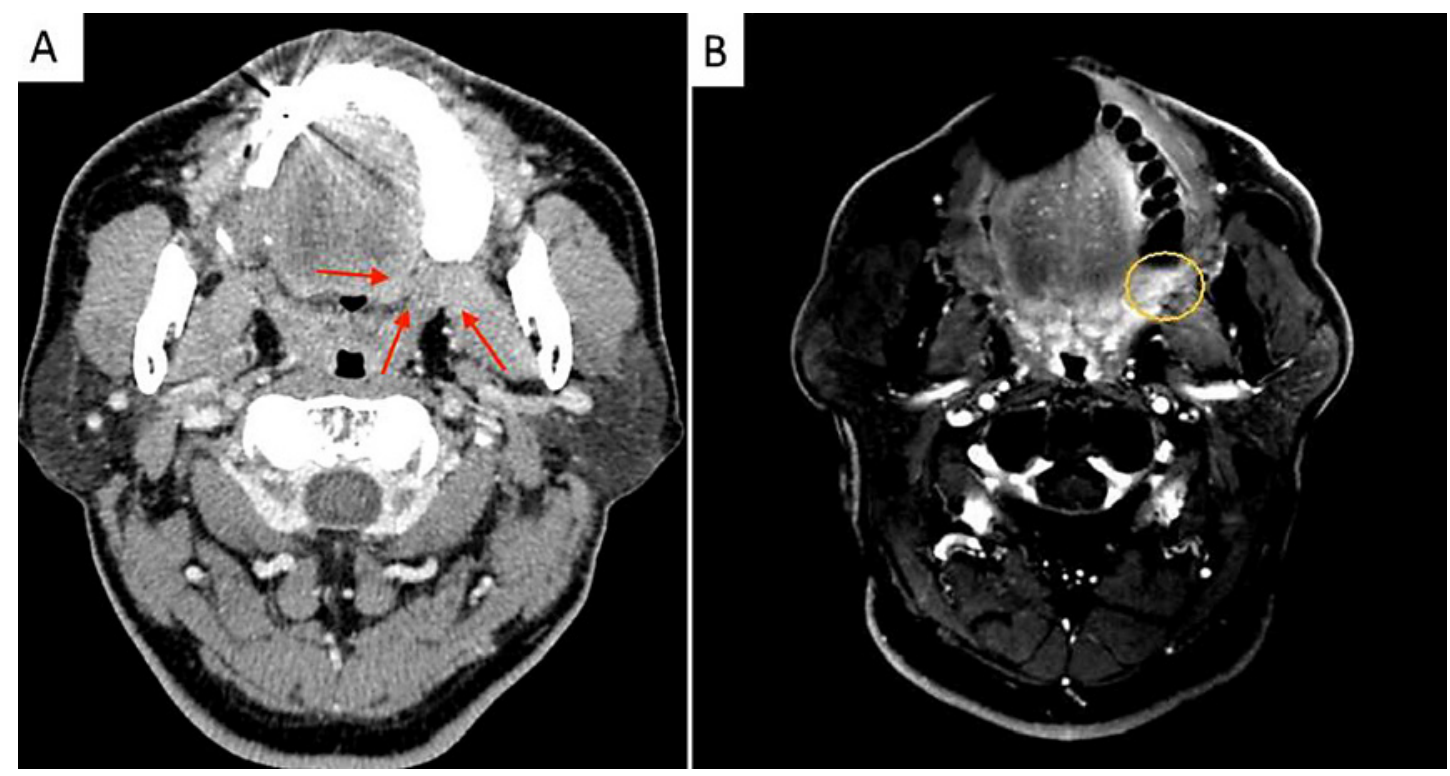

Fig. 1. Computed tomography (CT) (A) and magnetic resonance imaging (MRI) (B) identified a well-circumscribed, solid, oval-shaped mass lesion with a relatively clear margin in the left maxillary area. 

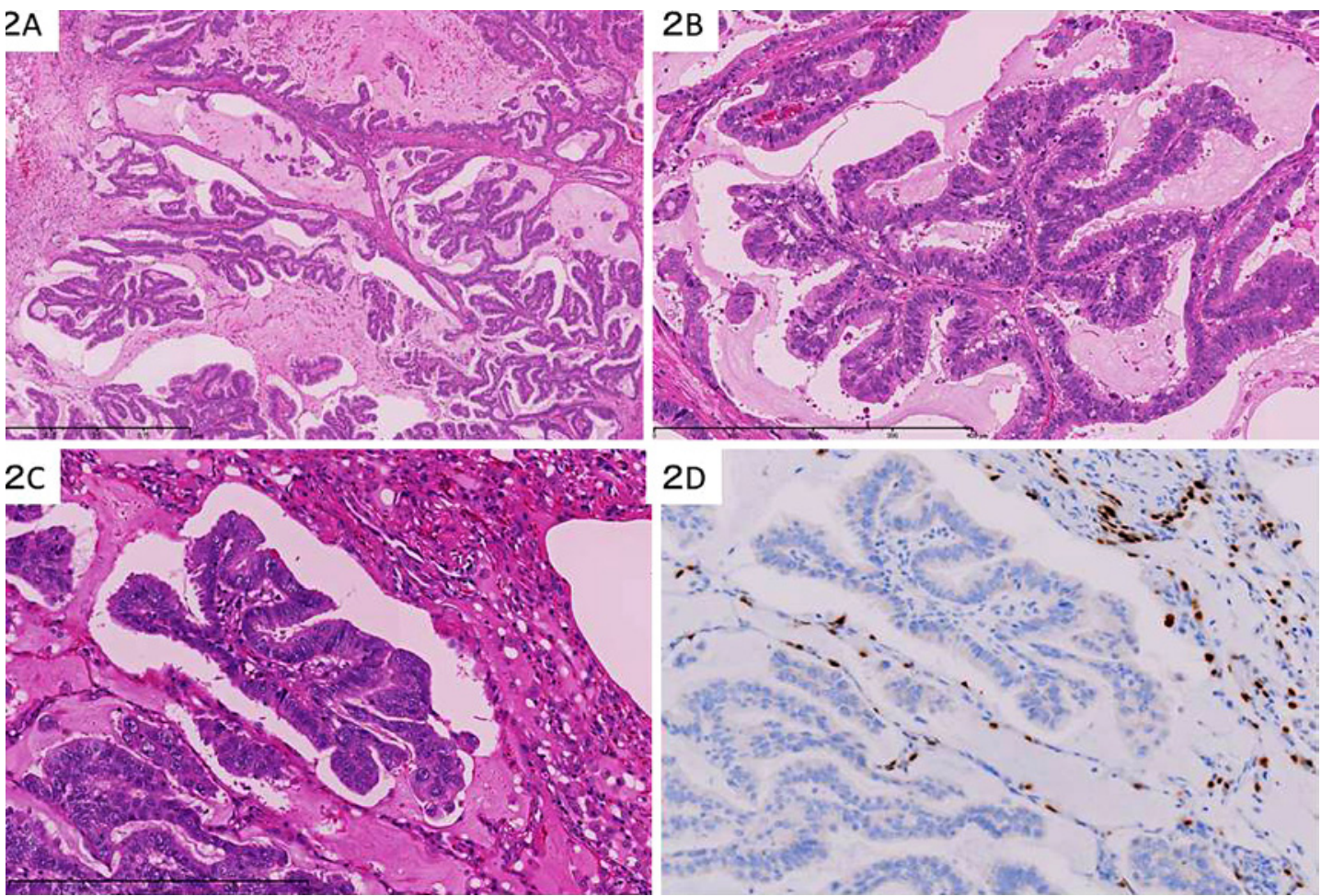

Fig. 2. Pathological findings from the resected maxillary tumor revealed that multiple and various sized cystic lumens filled up with solid parts, which were occupied by the papillary structures of the tumor cells $(\mathbf{A}, \times 5)$. The tumor cells had an oval nucleus and abundant eosinophilic cytoplasm $(\mathbf{B}, \times 20)$. Histological findings in the pulmonary nodule were similar with a previously resected maxillary gingiva tumor $(\mathbf{C}, \times 20)$. Immunological staining in resected pulmonary tumor was negative for TTF-1 (D, ×20).

mass and other organs. Based on these findings, submandibular sialoadenectomy with submandibular dissection (level I) was performed. Microscopically, the tumor showed a multicystic lumen filled with solid parts, which were occupied by papillary structures of the tumor cells (Fig. 2A). The nuclei were uniformly bland, and mitotic figures were rare (Fig. 2B). The tumor was encapsulated with a fibrous capsule and showed expansive growth. Some micropapillary nests of tumor cells were observed in the stroma and salivary gland around the tumor. Wide surgical excision was performed.

As pathological regional lymph node metastasis was detected ( $\mathrm{pN} 1)$, postoperative radiotherapy (70.9 Gy) was performed with periodic follow-up.

Three years later, abnormal findings were occasionally noted on chest radiographs during health screening. Chest CT revealed a nodule in the right lower lobe and mediastinal lymph node swelling (Fig. 3A, B). Thoracic surgery of the right lung nodule was performed and the histological findings were similar to the previously resected maxillary gingiva tumor (Fig. 2C). The resected pulmonary tumor was negative for thyroid transcription factor-1 (TTF-1) on immunohistochemical staining (Fig. 2D). We confirmed the diagnosis of pulmonary metastasis of papillary cystadenocarcinoma. Chemotherapy with cisplatin and 5-fluorouracil was performed with a 28-day cycle. However, four cycles of chemotherapy failed to reduce the mediastinal lymph node size. Subsequently, several chemotherapies including paclitaxel and nivolumab were performed; however, the mediastinal lymph node enlarged gradually and new metastatic pulmonary masses were presented over 3 years after these chemotherapies. 
A
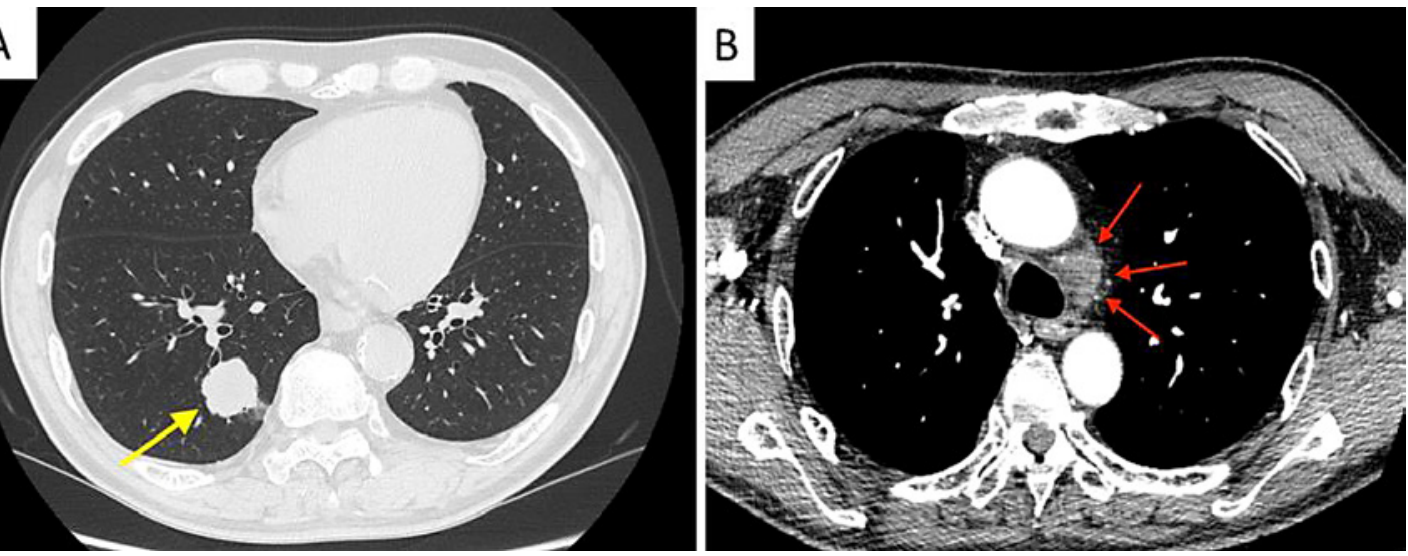

Fig. 3. Chest computed tomography (CT) showed right pulmonary nodule (A) and mediastinal lymph node swelling (B).

\section{Discussion}

Here, we reported a case of papillary cystadenocarcinoma arising from the maxilla that developed pulmonary metastasis 3 years after radical surgery of the primary tumor and regional lymph node.

Papillary cystadenocarcinoma shows low-grade histological and clinical features [1-3]. In a population-based study, the 5- and 10-year cause-specific survival rates were 97.0 and $81.4 \%$, respectively [12] and no patients with papillary cystadenocarcinoma of the salivary gland died of the disease. Therefore, papillary cystadenocarcinoma is considered to be a lowgrade malignancy according to the WHO classification and other reviews of the literature [1, 2]. Foss et al. [3] reported 40 cases of cystadenocarcinoma, 4 of which (10\%) presented with regional lymph node metastases, and $3(7.7 \%)$ showed local recurrence at an average of 76 months after initial treatment. In addition, there were some reports of a high-grade malignant variant that led to relatively high rates of metastasis for regional lymph node and recurrence with rapid progression [6-11]. These patients were rescued by surgery. However, these findings suggest that local recurrence [5] and cervical lymph node recurrence could be more common than previously thought in papillary cystadenocarcinoma [9-12]. In contrast to local recurrence [5] and/or regional lymph node metastasis, our case developed pulmonary metastasis, which is an extremely rare clinical manifestation during the clinical course of papillary cystadenocarcinoma. We searched the PubMed database using the keywords "cystadenocarcinoma," "metastasis," "distant metastasis," and "pulmonary metastasis," but we found no reports of cases that developed distant metastasis in other organs. Our experience indicated the possibility of distant metastasis in papillary cystadenocarcinoma. Therefore, we emphasize the importance of long-term follow-up and/or careful examination in patients with papillary cystadenocarcinoma.

This disease is more commonly observed in the major salivary glands (65\%) and occurs most often in the parotid gland (95\%) [1, 2]. Papillary cystadenocarcinoma arising from the sublingual glands is extremely rare and is found in the lip, buccal mucosa, palate, tongue, and retromolar trione [3-8]. Ectopic salivary gland tissue has been reported in a wide variety of locations, including the maxilla and mandible. Maxillary gingiva tumor as in the present case is extremely rare in minor salivary gland cystadenocarcinoma $[4,5,8]$. Pollet et al. [6] reported relatively high-grade papillary cystadenocarcinoma of the tongue with local and regional 
recurrence 6 months after partial glossectomy and bilateral neck dissection. Yamada et al. [7] also described high-grade papillary cystadenocarcinoma of the sublingual gland that showed recurrence. It remains unclear whether tumors originally located in the minor salivary glands, as in the present case, could show high-grade tumor biology compared to those from the major salivary glands. There are no defined pathological parameters for grading of salivary gland cystadenocarcinoma, but columnar cells with moderate atypia and brisk mitotic activity were reported to be correlated with recurrence and nodal metastasis [3]. The pathological findings in the present case were not always consistent with those reported as high-grade papillary cystadenocarcinoma [6-11].

Our case was treated with cisplatin plus 5-FU chemotherapy, but the tumor failed to show a significant reduction in size. Yamada et al. [7] described a case of advanced papillary cystadenocarcinoma that showed a significant response to combined chemotherapy with cisplatin and S-1 (a novel oral 5-FU antitumor drug) that combines three pharmacological agents: tegafur (a prodrug of 5-FU); 5-chloro-2,4-dihydroxypyridine (an inhibitor of dihydropyrimidine dehydrogenase); and potassium oxonate. Due to the rarity of this disease, the usefulness of chemotherapy for advanced and metastatic cystadenocarcinoma remained unclear. Additional clinical experience is required to evaluate the efficacy of chemotherapy in this disease.

In summary, the findings presented here suggest that papillary cystadenocarcinoma has the potential to produce distant metastasis during the clinical course. Based on our experience with this case, we emphasize that long-term follow-up and/or careful examination are necessary in patients with papillary cystadenocarcinoma, especially in those initially presenting with regional lymph node metastasis. This case and a review of the literature indicated that papillary cystadenocarcinoma of salivary gland origin exhibits a wide morphological spectrum.

\section{Statement of Ethics}

Ethical approval was relevant or applicable to this case report. Full informed consent was obtained from the patient to publish the manuscript and images .

\section{Disclosure Statement}

None of the authors have any relevant financial relationships with a commercial interest.

\section{Funding Sources}

None.

\section{Author Contributions}

Fumihiro Nishimaki, Takahiko Gibo, and Keita Tsukada operated and followed the patient, Takuro Noguchi, Toshirou Fukushima, Takashi Kobayashi, Nodoka Sekiguchi, Takesumi Ozawa, and Tomonobu Koizumi treated the patient with chemotherapy. Fumihiro Nishimaki and Tomonobu Koizumi drafted the manuscript. All authors contributed equally to this work and have read and approved the final manuscript. 


\section{References}

1 Histological classification of salivary gland tumors. 2nd ed. Berlin: Springer; 1991. p. 1-38.

2 Aloudah NM, Raddaoui E, Aldhahri S, Al-Abbadi MA. Low-grade papillary cystadenocarcinoma of the parotid gland: presentation of a case with cytological, histopathological, and immunohistochemical features and pertinent literature review. Diagn Cytopathol. 2009;37(2):128-31.

3 Foss RD, Ellis GL, Auclair PL. Salivary gland cystadenocarcinomas. A clinicopathologic study of 57 cases. Am J Surg Pathol. 1996;20(12):1440-7.

4 Wanjari SP, Wanjari PV, Patidar KA, Parwani RN. Papillary cystadenocarcinoma of the hard palate. BMJ Case Rep. 2014;2014:2014.

5 Srivanitchapoom C, Sittitrai P, Mahanupab P. Central papillary cystadenocarcinoma of the mandible: A case report and review of the literature. Int J Surg Case Rep. 2014;5(6):330-4.

6 Pollett A, Perez-Ordonez B, Jordan RC, Davidson MJ. High-grade papillary cystadenocarcinoma of the tongue. Histopathology. 1997;31(2):185-8.

7 Yamada S, Matsuo T, Baba N, Rokutanda S, Kawasaki G, Mizuno A, et al. High-grade papillary cystadenocarcinoma of the sublingual gland: a case report. J Oral Maxillofac Surg. 2007;65(6):1223-7.

8 Brookstone MS, Huvos AG. Central salivary gland tumors of the maxilla and mandible: a clinicopathologic study of 11 cases with an analysis of the literature. J Oral Maxillofac Surg. 1992;50(3):229-36.

9 Li Y, Li LJ, Huang J, Han B, Pan J. Central malignant salivary gland tumors of the jaw: retrospective clinical analysis of 22 cases. J Oral Maxillofac Surg. 2008;66(11):2247-53.

10 Cavalcante RB, da Costa Miguel MC, Souza Carvalho AC, Maia Nogueira RL, Batista de Souza L. Papillary cystadenocarcinoma: report of a case of high-grade histopathologic malignancy. Auris Nasus Larynx. 2007; 34(2):259-62.

11 Mukaigawa T, Hayashi R, Miyazaki M, Shinozaki T, Tomioka T, Fujii S. Cystadenocarcinoma of the salivary glands with potential lymph node metastasis. Auris Nasus Larynx. 2016;43(3):340-4.

12 Cai CF, Sun JY, He ZY, Lin HX, Liu FR, Wang Y. Clinicopathological characteristics, treatment, and survival outcomes of cystadenocarcinoma of the salivary gland: a population-based study. Onco Targets Ther. 2016; 9:6569-72. 